\title{
Radio Frequency Based Emergency Exit Node Technology
}

\author{
Youngwoo Choi', Dong Kyoo Kim ${ }^{1 \dagger}$, Do Wook Kang ${ }^{1}$, Wan Sik Choi ${ }^{2}$ \\ ${ }^{1}$ Cooperative Vehicle-Infra Research Section, Electronics and Telecommunications Research Institute 218 Gajeongno, Yuseong-gu, \\ Daejeon 305-700, Korea \\ ${ }^{2}$ Autonomous Driving System Research Section, Electronics and Telecommunications Research Institute 218 Gajeongno, Yuseong- \\ gu, Daejeon 305-700, Korea
}

\begin{abstract}
This paper introduces an indoor sensor fusion wireless communication device which provides the Location Based Service (LBS) using fire prevention facility. The proposed system can provide information in real time by optimizing the hardware of WiFi technology. The proposed system can be applied to a fire prevention facility (i.e., emergency exit) and provide information such as escape way, emergency exit location, and accident alarm to smart phone users, dedicated terminal holders, or other related organizations including guardians, which makes them respond instantly with lifesaving, emergency mobilization, etc. Also, the proposed system can be used as a composite fire detection sensor node with additional fire and motion detect sensors.
\end{abstract}

Keywords: LBS, WLAN, safety, positioning, platform

\section{INTRODUCTION}

An indoor sensor fusion localization system using fire facility utilizes an indoor fire facility as LBS infra, and can provide the position and emergency information (crime, missing, disaster, etc.) of dedicated terminal holders or smart phone users at indoor and outdoor locations to the guardians or related organizations (police and National Emergency Management Agency) by applying localization node to the fire prevention infra such as emergency guide light and fire detector. Also, the system is capable of indoor localization with an error range of several meters.

Various attempts have been made regarding the domestic localization methods, but the GPS is not capable of indoor localization and the mobile communication base station Cell-ID method has low localization accuracy (Kim et al. 2012). Also, even though precise localization is necessary indoors or in large underground space, the localization

Received Apr 01, 2013 Revised Apr 23, 2013 Accepted Apr 29, 2013

tCorresponding Author

E-mail: kdk3606@etri.re.kr

Tel: +82-42-860-5620 Fax: +82-42-860-1085 infra is lacking. As for the localization technique that uses the existing WLAN infra indoors, the localization precision has large variation depending on measuring point because the AP installation environment is not constant. In the case of WLAN AP for wireless communication, the localization precision is hard to be improved by densely installing AP due to the self-interference phenomenon (Kim \& Choi 2011, Hong 2009, Ergin 2007).

As shown in Fig. 1, the indoor sensor fusion localization system proposed in this paper integrates localization node into the fire facilities that are densely installed indoors, and can provide the service which informs the evacuation route via smart phone or dedicated terminal (hereinafter referred to as smart phone) in case of disaster including fire by locating the position of smart phone that is within the communication radius of node through the communication between the installed node and smart phone. For this purpose, the localization node should not interfere with the existing WLAN AP for wireless communication and the WLAN AP also should not interfere with the localization node, which requires appropriate cell planning and interference avoidance technique (Kim 2006, Choi et al. 2011, Huang et al. 2000). 


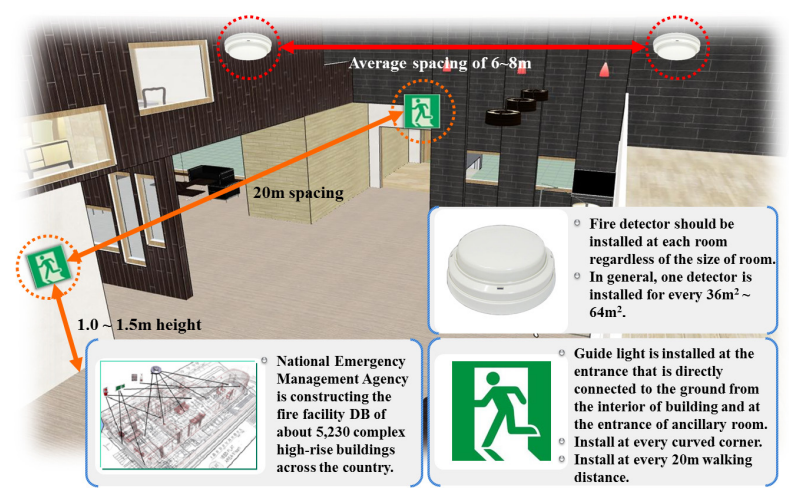

Fig. 1. Indoor fire prevention facility for LBS infra.

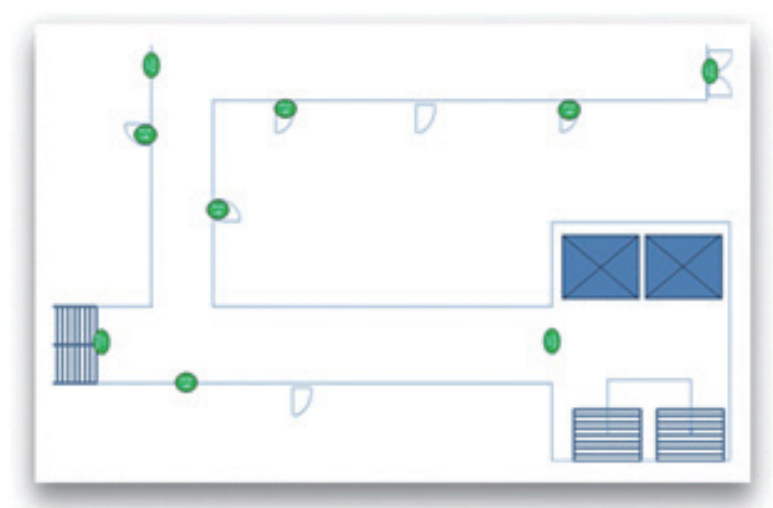

Fig. 2. Node installation.

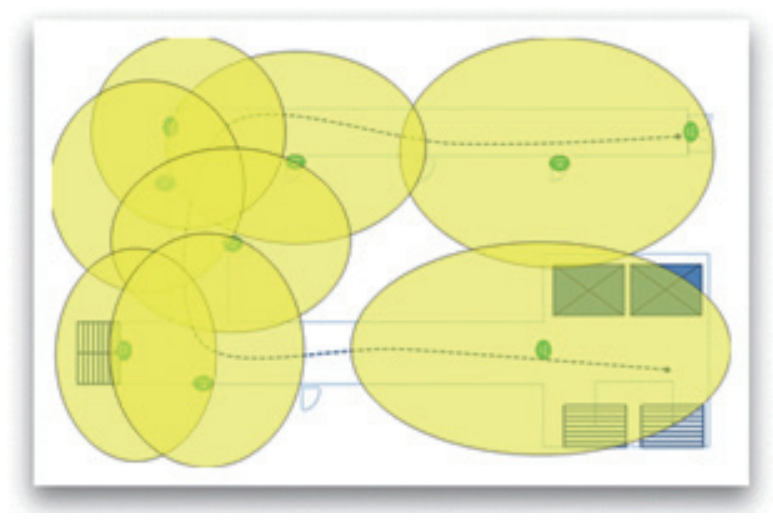

Fig. 3. Before cell planning.

Fig. 2 is an example of the node installation. When the cell planning is not used as shown in Fig. 3, it is hard to figure out whether the smart phone is located within the communication radius of certain node, and a number of nodes simultaneously perceive the smart phone, which decreases the localization accuracy. On the other hand, when the cell planning is used as shown in Fig. 4, as there could be shadow areas, the position or moving route of user can be estimated by integrating the sensor information

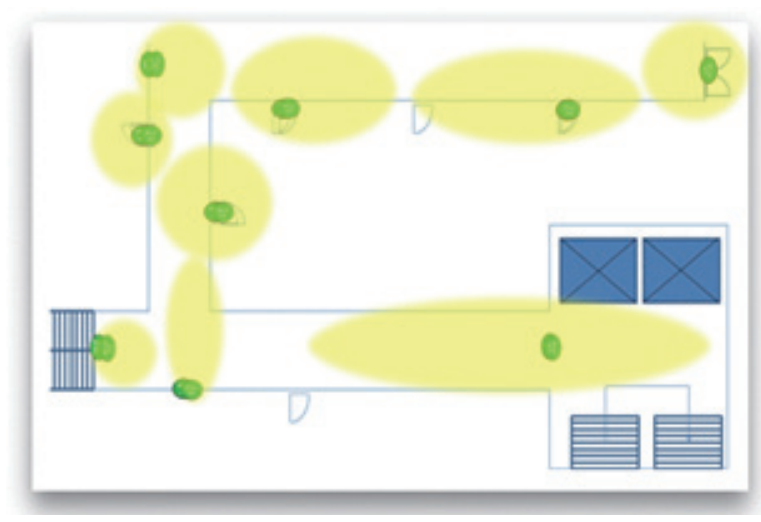

Fig. 4. After cell planning.

embedded in the smart phone. The indoor sensor fusion localization node proposed in this paper uses the cell planning and interference avoidance technique, and has a much lower possibility of colliding with WLAN AP for wireless communication compared to the existing AP. Thus, a number of nodes can be installed and more precise localization is possible.

\section{MAIN SUBJECT}

\subsection{Positioning Information Embedded Beacon Technology}

In the localization node of indoor sensor fusion localization system proposed in this paper, the MAC Address and Service Set IDentification (SSID) are used for the device identifier at the IEEE 802.11 standard. Among these, the MAC Address is an inherent 48-bit value that is assigned when the device is produced, and is mainly used as a link layer identifier of network. In general, the SSID is preferred for device identification at the WLAN application layer, and in the case of WLAN, the SSID is used for the identification of Access Point (AP). For the SSID, an expression with linguistic meaning is commonly used which ranges from a word such as company name or ID to a sentence. This is to make it easier for users to directly search, select, and access the device. Therefore, the SSID consists of ASCII character. The SSID which consists of linguistic term has an advantage of good visibility when users search/recognize the device, but has poor applicability except for that.

On the other hand, in the localization field, when collecting adjacent WLAN AP information for analyzing the RF characteristic of specific position, the SSID is used to distinguish the collected APs. This is using the SSID in the same level as using the SSID when users search device in the WLAN. In the IEEE 802.11 standard, the SSID can 


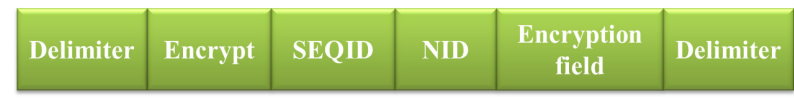

Fig. 5. SSID format for positioning beacon.

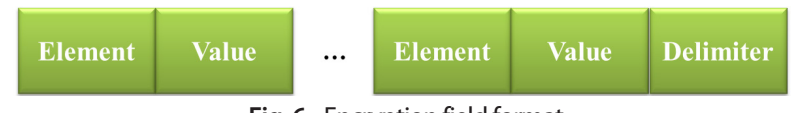

Fig. 6. Encryption field format.

Table 1. Positioning beacon element-value mapping table.

\begin{tabular}{|c|c|c|c|c|}
\hline \multirow[b]{2}{*}{ Element } & \multicolumn{4}{|c|}{ Value } \\
\hline & Size & Value description & Value range & $\begin{array}{l}\text { Conversion } \\
\text { method }\end{array}$ \\
\hline A & 1B & Transmit power value & $-72 \sim 20 \mathrm{dBm}$ & value +106 \\
\hline B & 1B & Antenna Gain & $0 \sim 35 \mathrm{dBi}$ & value +34 \\
\hline $\mathrm{C}$ & 1B & Antenna Type & $\begin{array}{l}\text { 0:omni, } 1 \text { :dir-15, 2:dir- } \\
\text { 30, 3:dir-60, 4:dir-90, } \\
\text { 5:patch, 6:others }\end{array}$ & value +34 \\
\hline $\mathrm{D}$ & 1B & $\begin{array}{l}\text { Antenna installation } \\
\text { azimuth }\end{array}$ & $\begin{array}{l}5 \text { degree, } 10 \text { degree, } \ldots \\
360 \text { degree }\end{array}$ & Value $/ 5+33$ \\
\hline E & & $\begin{array}{l}\text { Geospatial information } \\
\text { classification }\end{array}$ & - & value +34 \\
\hline $\mathrm{F}$ & & $\begin{array}{l}\text { Geospatial information } \\
\text { details }\end{array}$ & - & value +34 \\
\hline a & 3B & $\begin{array}{l}\text { Accelerometer value } \\
\text { deviation } \\
(\mathrm{x}, \mathrm{y}, \mathrm{z})\end{array}$ & $-1 \mathrm{~g} \sim 1 \mathrm{~g}$ & $\begin{array}{l}\text { Floor(Value +1) } \\
\quad * 46)+34\end{array}$ \\
\hline $\mathrm{b}$ & 1B & Battery life & $0 \%, 5 \%, \ldots, 100 \%$ & Value $/ 5+34$ \\
\hline $\mathrm{c}$ & 1B & Temperature & -46 degree $\sim 46$ degree & value +80 \\
\hline
\end{tabular}

be transmitted by loading onto beacon frame and probe response frame, and a maximum 32Byte of string can be used. In this regard, there is no restriction on the type of string, but it is common to use the ASCII code. In other words, when a string other than ASCII code is used, the possibility of recognition is not guaranteed. Therefore, to maintain compatibility, the ASCII code set needs to be used for the SSID. In this paper, the SSID format was defined as shown in Fig. 5 to store diverse information in the SSID using ASCII code set, and the beacon which transmits the SSID of this format was defined as positioning beacon.

The delimiter for the recognition of positioning beacon SSID is a 1Byte value and has 0x23 (\# character as ASCII code) value. Total 3 delimiters exist in the SSID (two in Fig. 5 and one in Fig. 6). The encrypt is a 1Byte value and represents the encryption type of encryption field. The SEQID is a 1Byte value and represents whether it is fragmented and the fragmentation order. The NID is a 4Bytes value and represents the ID of device which transmits the positioning beacon SSID.

As shown in Fig. 6, the encryption field of SSID format consists of numerous element-value pairs and the delimiter. The element-value pair is an information transfer system, and the element has the information type and the value has the information value, which are defined beforehand and then used. The element-value pair of positioning beacon was defined as shown in Table 1. In Table 1, each element should determine the final value using the conversion method for its own value.

The use of each element is as follows.

- Elements A and B : The transmit power value and antenna gain can be used by the terminal to calculate the distance loss between the positioning beacon device and terminal.

- Element $\mathrm{C}$ : The antenna type can be used by the terminal to determine the weight on the use of direction information from the positioning beacon.

- Element D : The antenna installation azimuth represents the azimuth that the front of antenna faces. The terminal can estimate its own moving direction using the antenna type and antenna installation azimuth, and they can be used as the correction information of digital compass sensor indoors.

- Elements E and F: The geospatial information classification and details are described below.

- Element a : The accelerometer value deviation can be used to provide the information on the position change of the installed antenna.

- Elements b and c : The battery life and temperature are the examples of the status information of positioning beacon device, and diverse status information can be defined and used.

In Table 1, the geospatial information, which is expressed in elements $\mathrm{E}$ and $\mathrm{F}$, can be used to decide how much to rely on the diverse sensor collected information including the RF signal that is used when the terminal performs the localization. The geospatial information consists of classification element and details element. The classification element is used to classify the type of space for each class, and the details element is used to represent the detailed spatial characteristic of the relevant class. Table 2 shows the examples for the definition of geospatial information.

\subsection{Software and Hardware of Indoor Sensor Fusion Localization System}

\subsubsection{Software Structure of Indoor Sensor Fusion Localization System}

Fig. 7 shows the software structure of cell planning which is necessary for the case when numerous positioning beacon devices installed within specific space simultaneously transmit the positioning beacons. The cell planning software consists of the beacon transmit and receive unit which 
Table 2. An example of the geospatial information classification.

\begin{tabular}{|c|c|c|c|c|c|}
\hline Classification & Corridor & Lobby & $\begin{array}{l}\text { Passage for } \\
\text { upper and } \\
\text { lower floors }\end{array}$ & Entrance & Others \\
\hline \multirow{6}{*}{ Details } & I - type & Small size & Stairs & $\begin{array}{l}\text { Building } \\
\text { entrance }\end{array}$ & \multirow{6}{*}{ Others } \\
\hline & $\neg$ - type & Medium size & Elevator & $\begin{array}{l}\text { Room } \\
\text { entrance }\end{array}$ & \\
\hline & $\mathrm{T}$ - type & Large size & Escalator & $\begin{array}{l}\text { Stairs } \\
\text { entrance }\end{array}$ & \\
\hline & +- type & \multirow{3}{*}{ Others } & $\begin{array}{l}\text { Moving } \\
\text { sidewalk }\end{array}$ & \multirow{3}{*}{ Others } & \\
\hline & $Y$ - type & & \multirow{2}{*}{ Others } & & \\
\hline & Others & & & & \\
\hline
\end{tabular}

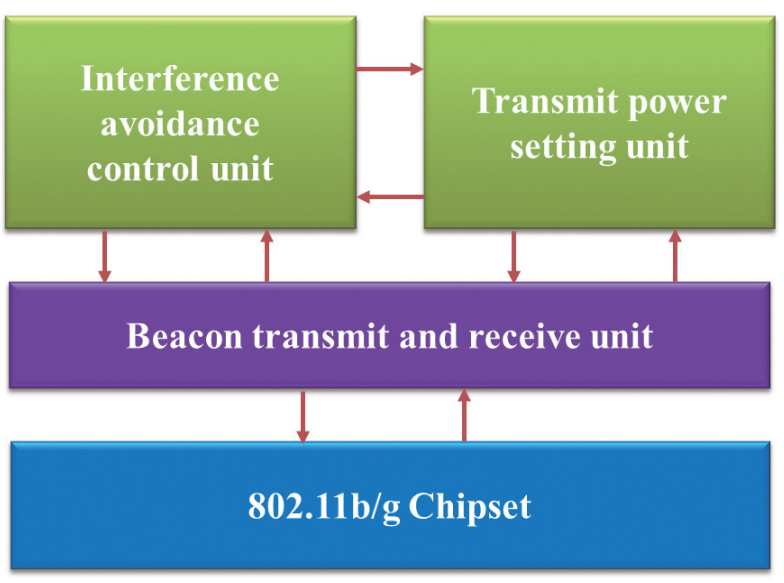

Fig. 7. The software structure of cell planning method.

transmits and receives the beacon signal by controlling $802.11 \mathrm{~b} / \mathrm{g}$ chipset, the transmit power setting unit which determines the transmit power of positioning beacon for transmission, and interference avoidance control unit which sets the channel of positioning beacon for transmission.

\section{(1) Structure of Transmit Power Setting Unit}

Fig. 8 shows the structure of transmit power setting unit. The SSID receive unit receives and stores the SSID of beacon which is received at the beacon receive unit through scanning process. The SSID parsing unit distinguishes the positioning beacon by parsing the stored SSID, and stores the information by parsing this. The path loss measurement unit measures the path loss of the scanned positioning beacon device based on the parsed information. The transmit power determining unit determines its own positioning beacon transmit power using the measured path loss information.

\section{(2) Structure of Interference Avoidance Control Unit}

Fig. 9 shows the structure of interference avoidance

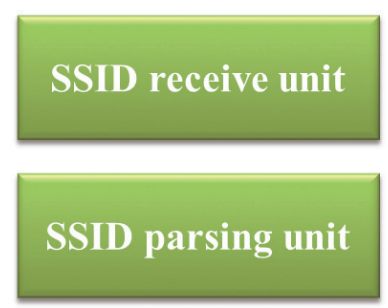

Path loss measurement unit

Transmit power determining unit

Fig. 8. The structure of transmit power setting block.

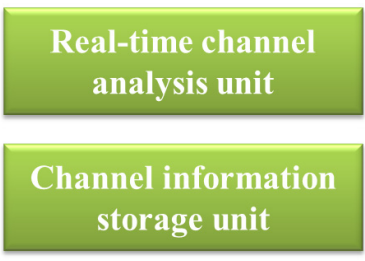

Avoidance channel management unit

Channel determining unit

Fig. 9. The structure of interference avoidance control block

control unit. The real-time channel analysis unit analyzes the status of channel that exists within the operating band (e.g., 2.4 GHz ISM band) in real time. The avoidance channel management unit sets/manages the channel to be avoided. The channel information storage unit deduces and stores the status and availability of entire channels using the channel status and avoidance channel information which are analyzed in real time. The channel determining unit determines the channel that has the highest interference avoidance effect based on the data stored in the channel information storage unit, and sets this as the channel for positioning beacon transmission.

\subsubsection{Hardware of Indoor Sensor Fusion Localization System}

The wireless transceiver module, which is applied to the node of indoor sensor fusion localization system, follows the IEEE 802.11b standard and supports a speed of up to $11 \mathrm{Mbps}$ at the wireless interface. Also, it is capable of ultralow power operation by dynamic power management, and can communicate with a smart phone without a separate AP through the limited AP mode which can limitedly perform the role of AP. As shown by the hardware architecture of wireless transceiver module in Fig. 10, the interior is loaded with a dual ARM7 processor. The first processor is the WLAN CPU and is equipped with WLAN software, and the second processor is the application CPU and is equipped with networking software. Also, the module is loaded with the interfaces such as SPI, UART, GPIO, and I2C for interfacing with external sensor or memory, and the embedded Real Time Clock (RTC) which can be operated with a battery.

Fig. 11 shows the implemented transceiver module, 


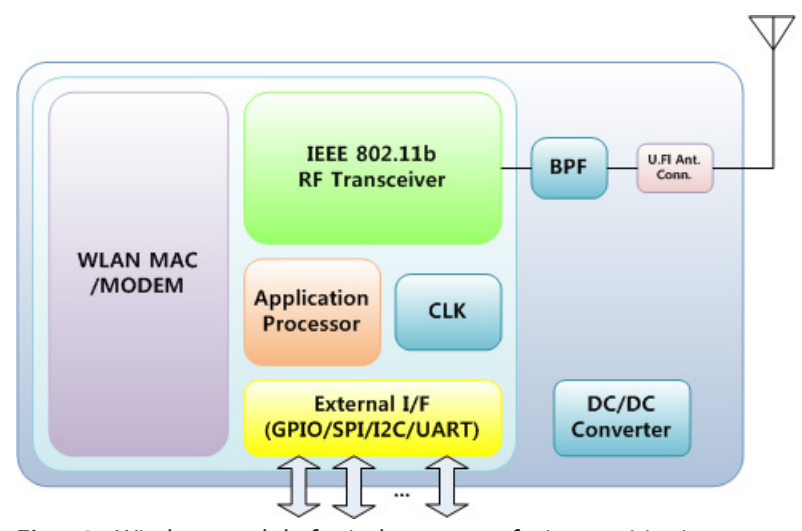

Fig. 10. Wireless module for indoor sensor fusion positioning system architecture.

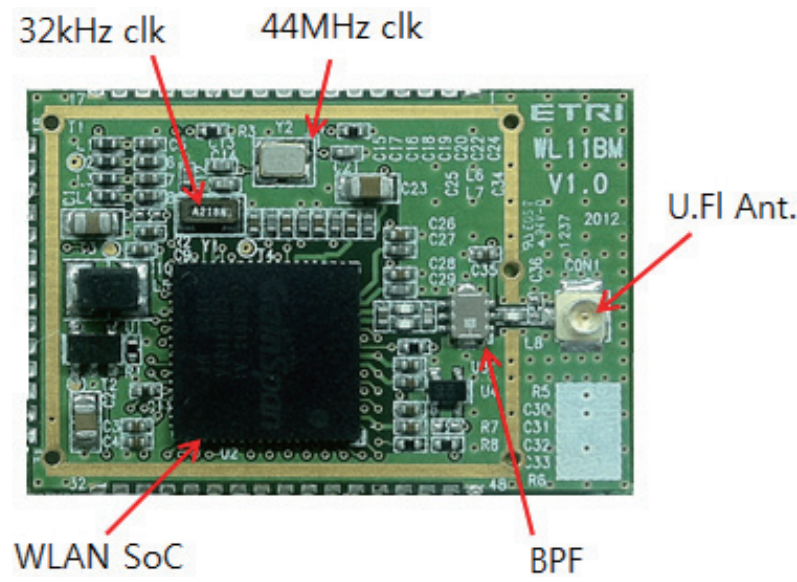

Fig. 11. Indoor sensor fusion positioning transceiver module.

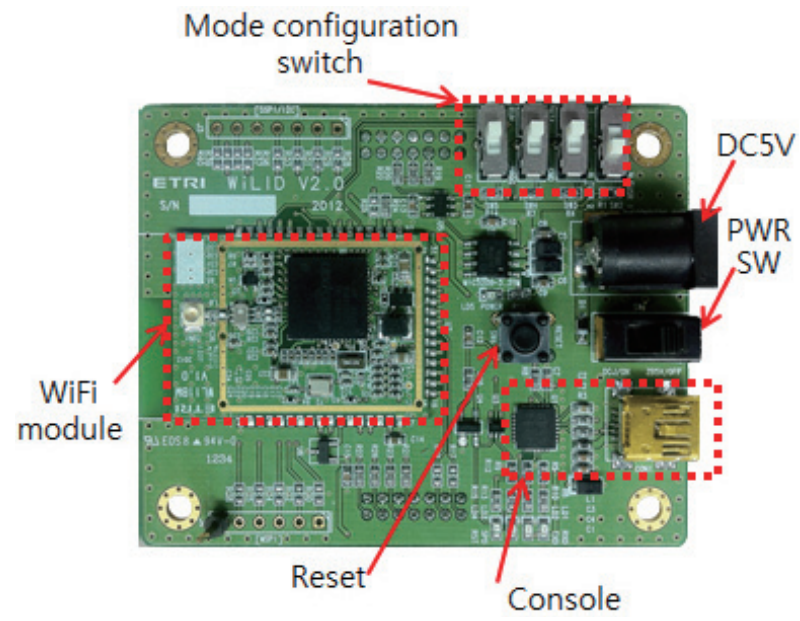

Fig. 12. Indoor sensor fusion positioning system.

and Fig. 12 shows the hardware of indoor sensor fusion localization node which is loaded with the transceiver module.

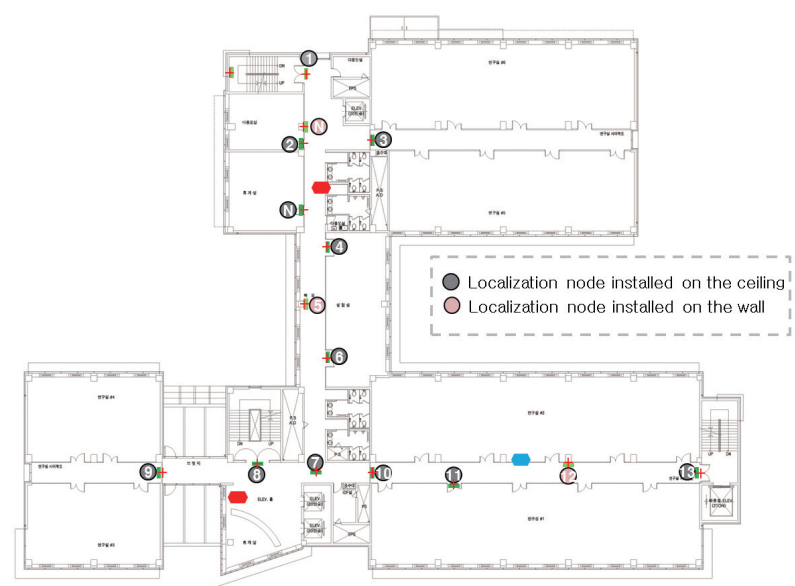

Fig. 13. Node installation drawing in the test bed.

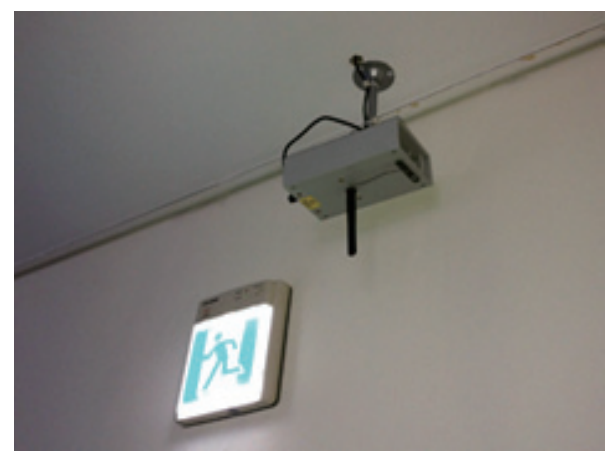

Fig. 14. Node for the ceiling.

\section{EXPERIMENT}

For applying 13 indoor sensor fusion localization nodes to the test bed as shown in Fig. 13 and performing an experiment for the cell planning algorithm and interference avoidance algorithm using a smart phone, it was assumed that the nodes are installed at the emergency guide light in the building. The experiment was carried out after installing the nodes on the ceiling and wall as shown in Figs. 14 and 15. When the power is applied to each node after setting the transmit power value as a maximum, every node scans the adjacent node. Based on this result, each node performs the cell planning algorithm and then adjusts the transmit power to an appropriate value for itself.

\subsection{Cell planning algorithm test}

Fig. 16 shows the proposed cell planning algorithm. Each node scans the SSID of adjacent node, extracts the individual coordinate of the scanned nodes, calculates the straight-line distance to the corresponding node, extracts 


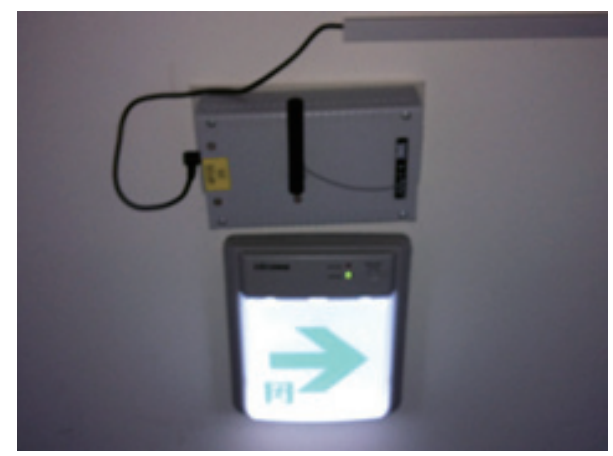

Fig. 15. Node for the wall.

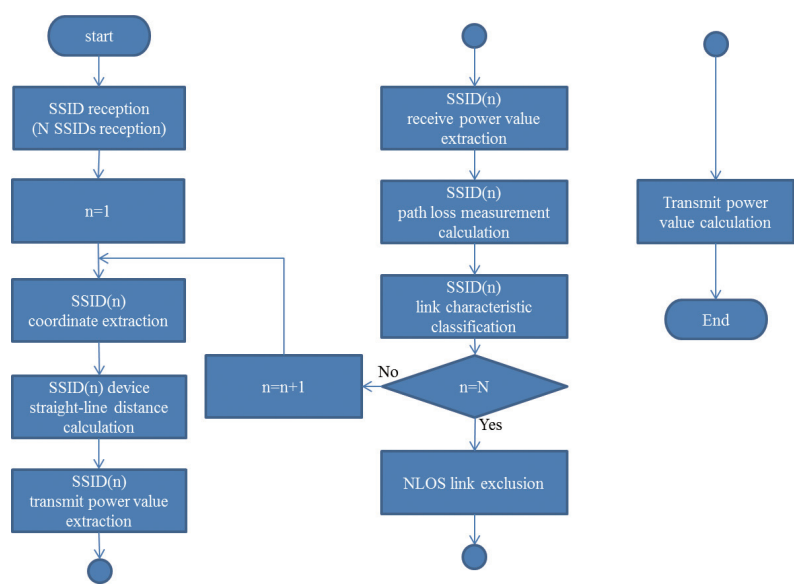

Fig. 16. Flowchart of cell planning algorithm.

the transmit power value and receive power value, and calculates the path loss measurement. It classifies the link characteristic based on this result, excludes the NLoS (Non Line-of-Sight) link, adjusts the beacon transmit power value, and transmits the beacon.

For the cell planning algorithm test, the localization node was loaded with a web server, and the data transmitted from the adjacent node was collected. Fig. 17 shows an example of the execution of web software for adjacent node data collection.

The initial transmit power of every localization node was set to be $9 \mathrm{dBm}$, and Table 3 shows the transmit power of each node before and after the application of cell planning algorithm. After the application of algorithm, the transmit power of node decreases depending on the status of adjacent node. Thus, when the received signal strength of adjacent nodes at each node, which is measured by accessing the web server of each node, is presented in a graph, it is as shown in Fig. 18. The Node 12 was excluded from the experiment data due to the operation error. In the graph of Fig. 18, the initial value is the received signal power value of adjacent node collected before the application of

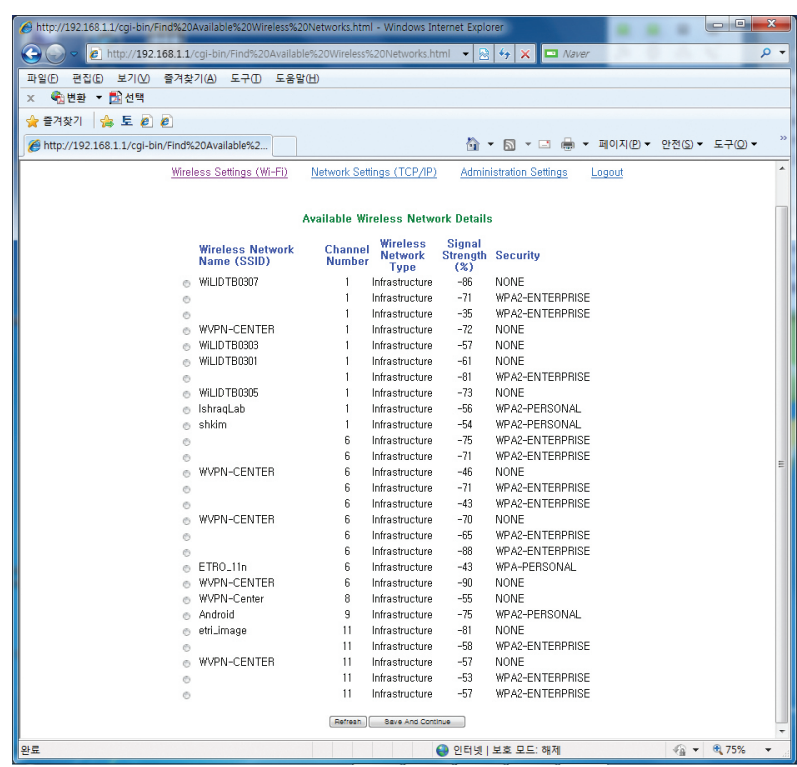

Fig. 17. Example of web screen for collecting signal strength scan results.

Table 3. Results of cell planning.

\begin{tabular}{ccc}
\hline Node number & Before the application $(\mathbf{d B m})$ & After the application $(\mathbf{d B m})$ \\
\hline 1 & 9 & -1 \\
2 & 9 & -2 \\
3 & 9 & -1 \\
4 & 9 & -2 \\
5 & 9 & -7 \\
6 & 9 & -8 \\
7 & 9 & -5 \\
8 & 9 & -3 \\
9 & 9 & -4 \\
10 & 9 & -11 \\
11 & 9 & -11 \\
12 & 9 & -10 \\
13 & 9 & -6 \\
\hline
\end{tabular}

cell planning algorithm. The received signal power value of adjacent node, collected after the cell planning algorithm is applied to each node once, is expressed as the primary planning. From the graph, it is shown that the received signal strength of adjacent node is generally decreased by the application of cell planning algorithm. This phenomenon is the result of power distribution adjustment between each node. In other words, when there are many adjacent nodes which have a high received power value, the overlapping of power distribution is high. And in the opposite case, the overlapping of power distribution is low, which improves the level of separation.

The result of power distribution adjustment can be examined more clearly by combining the graph for each node in Fig. 18. The comparison graph for the objective evaluation of cell planning algorithm is shown in Fig. 19. 

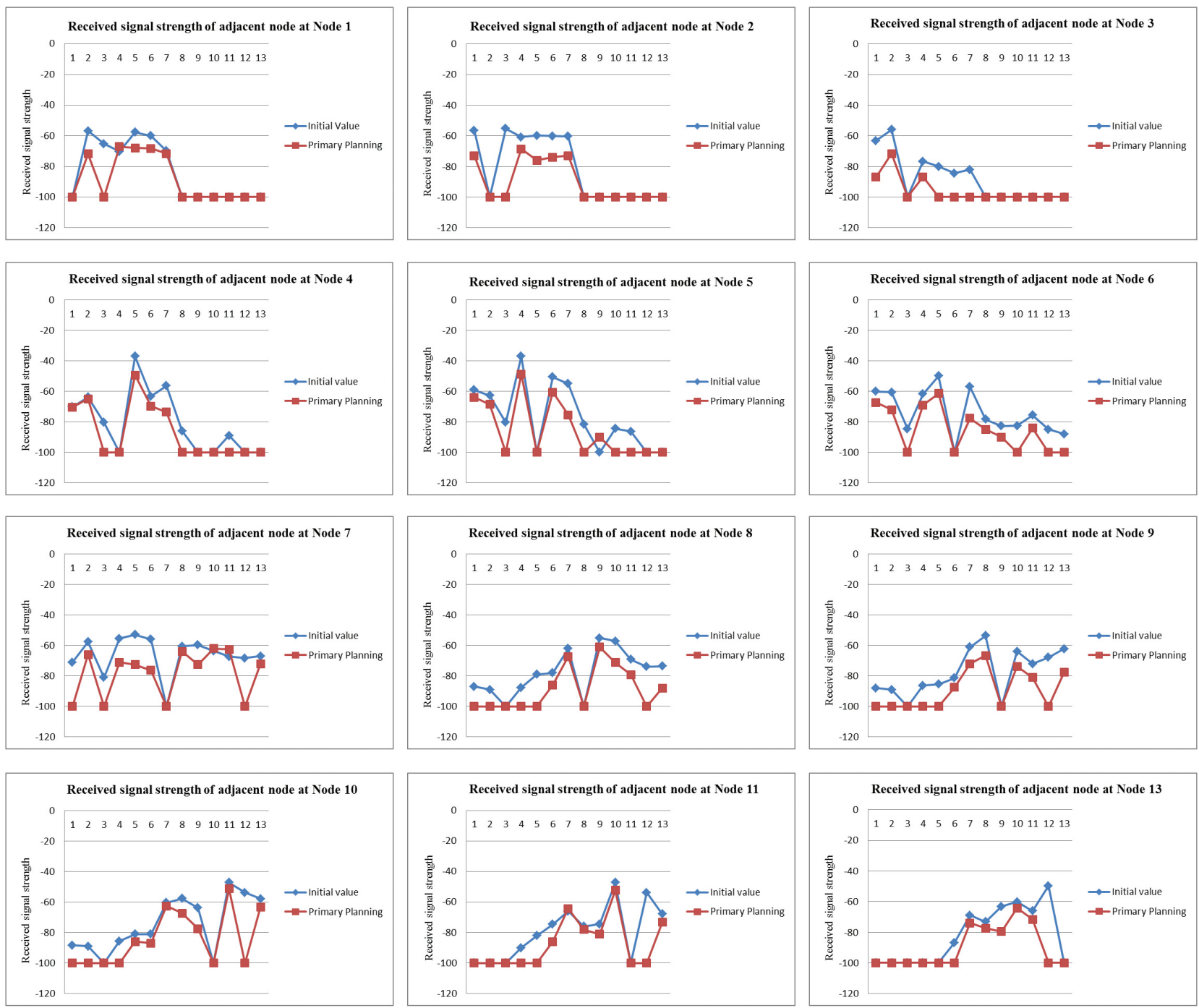

Fig. 18. Received signal strength of adjacent node at each node.

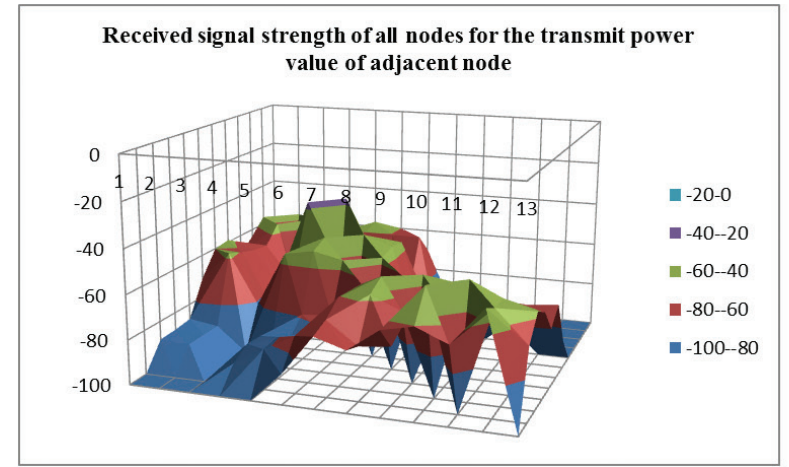

(a) Before the application of cell planning algorithm

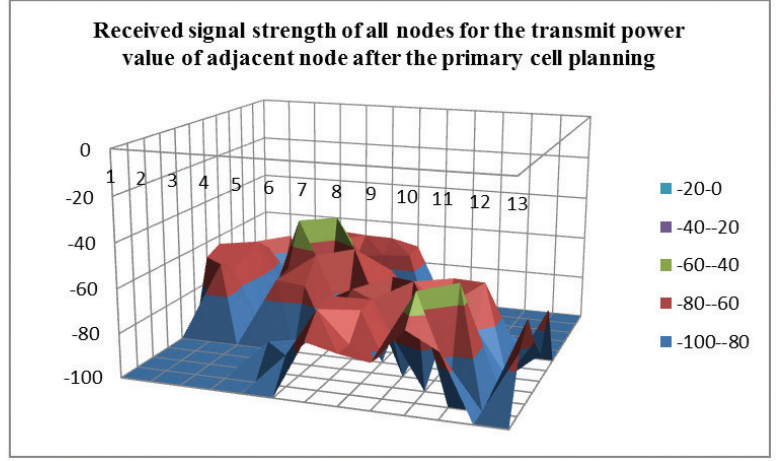

(b) After the application of cell planning algorithm

Fig. 19. Received signal strength of all nodes before and after the application of cell planning algorithm.

Fig. 19a shows the transmit power distribution which was measured at the same condition as general WLAN AP (i.e., Channels 1, 5, and 10 are used so that it has the maximum transmit output value and that there is no overlapping between channels). Fig. 19b shows the transmit power distribution when the cell planning algorithm is applied. 


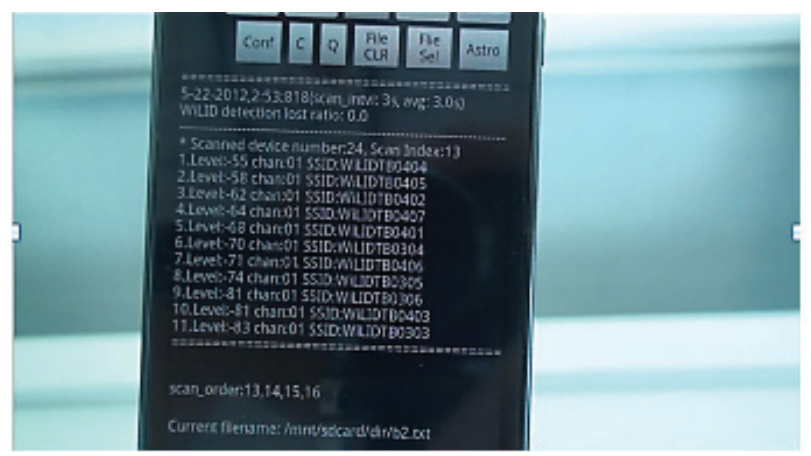

(a) Before the application of cell planning algorithm

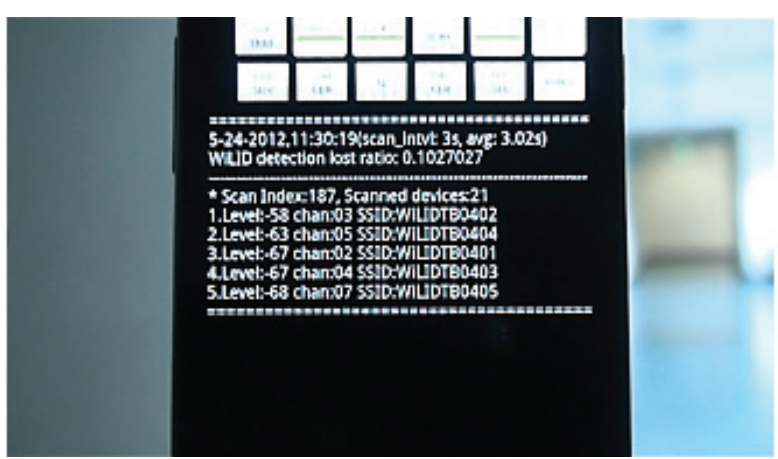

(b) After the application of cell planning algorithm Fig. 20. Received signal strength of all nodes before and after the application of cell planning algorithm.

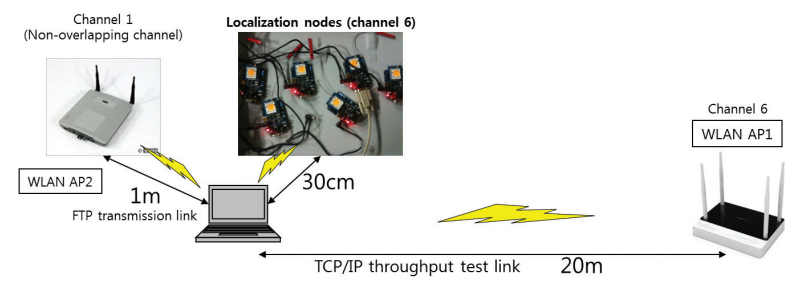

Fig. 21. Inter-channel interference test configuration.

In Fig. 19, $x$-axis represents the index of each node, $y$-axis represents the index of adjacent node for each node of $\mathrm{x}$-axis, and $\mathrm{z}$-axis represents the received signal strength. When the z-axis values before and after the application of cell planning algorithm are compared, the $\mathrm{z}$-axis value is generally lower after the application of algorithm. Therefore, it can be easily shown that there is overall improvement in the separation level of power distribution. This indicates that when a smart phone collects the signal of localization node to locate its own position, the localization is hard to be achieved before the application of cell planning algorithm as many localization nodes which have strong signal strength are scanned as shown in Fig. 20a, but the localization is easy to be achieved after the application of cell planning algorithm as only the nodes which are adjacent to the smart phone are scanned as shown in Fig. 20b.

\subsection{Interference avoidance algorithm test}

The algorithm test for avoiding the interference between the localization node and the WLAN AP for wireless communication was carried out as follows. First, an underground storage, which is isolated from radio waves, was selected as a test site to increase the reliability of interference test. The test setup is shown in Fig. 21, where the laptop establishes a TCP/IP throughput test link with WLAN AP1 and a FTP transmission link with the WLAN AP2. The TCP/IP throughput test link is set to the channel 6 , and the FTP transmission link set to the channel 1. WLAN AP2 and a bunch of localization nodes are placed adjacent to the laptop to make interference against the TCP/IP throughput test link of the far-off WLAN AP1. In this test, we can evaluate how much the TCP/IP link performance is deteriorated by the interferers.

The throughput test for the TCP/IP test link was performed. As shown in Fig. 22, the WLAN AP2 makes nonoverlapping interference against the TCP/IP test link. The
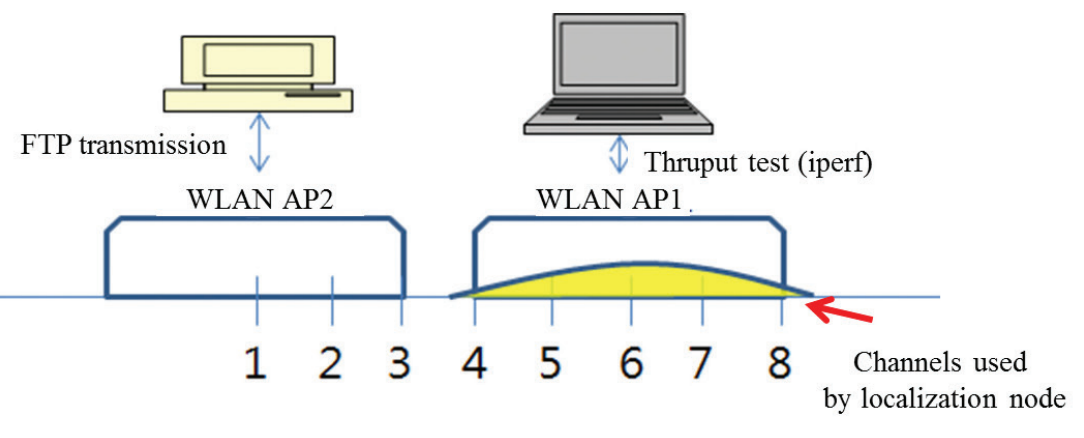

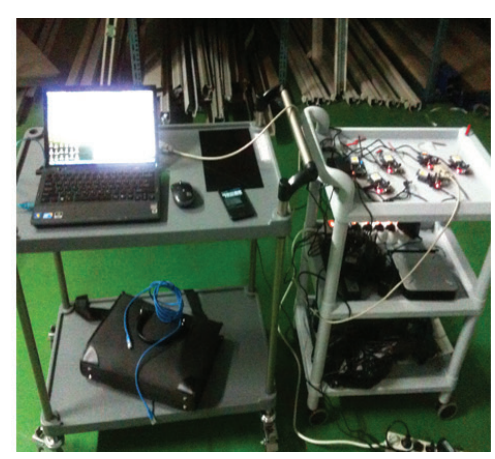

Fig. 22. Channel setting for inter-channel interference test. 
Table 4. Test results of interference avoidance between wireless channels.

\begin{tabular}{lcc}
\hline & $\begin{array}{c}\text { TCP data capacity that is } \\
\text { successfully transferred } \\
\text { (TCP transfer) (MBytes) }\end{array}$ & $\begin{array}{c}\text { TCP } \\
\text { bandwidth(Mbps) }\end{array}$ \\
\hline $\begin{array}{l}\text { (Noninterference } \\
\text { condition) }\end{array}$ & $(22.2)$ & $(18.6)$ \\
\hline $\begin{array}{l}\text { Before the application of } \\
\text { interference avoidance } \\
\text { algorithm }\end{array}$ & 11.1 & 9.28 \\
\hline $\begin{array}{l}\text { After the application of } \\
\text { interference avoidance } \\
\text { algorithm }\end{array}$ & 18.8 & 15.7 \\
\hline
\end{tabular}

adjacent localization nodes are set to channel 6 that makes overlapping interferences against the TCP/IP test link when the nodes transmit beacons.

Regarding the TCP/IP throughput test of the laptop that is connected with WLAN AP1, Table 4 shows the test results when the power of every localization node is turned off (noninterference condition), when the power of every localization node is turned on and the channel interference avoidance algorithm is not applied, and when the power of every localization node is turned on and the channel interference avoidance algorithm is applied. Based on the TCP data capacity that is successfully transferred at noninterference condition, as shown in the test results, when the interference avoidance algorithm is applied, the effect of interference on the WLAN AP for wireless communication induced by localization node decreases, which in turn increases the TCP transfer.

\section{CONCLUSION}

The indoor sensor fusion localization system using fire facility can greatly improve the localization precision compared to the existing indoor localization method which has a localization error of more than ten meters and does not guarantee enough precision to discern floor information. Also, the system has a much lower possibility of interference than the existing AP as it applied the channel interference avoidance technique to the existing AP for wireless communication. Therefore, the localization node can be installed densely, and this infra expansion enables more precise localization.

\section{ACKNOWLEDGMENTS}

This research was funded by the Ministry of Knowledge Economy with the Global Specialized Technology Development Project, 'Development of Fire Prevention
Facility-based Indoor/Outdoor Sensor Fusion Positioning Device and LBS Service Platform.

\section{REFERENCES}

Kim, D. K., Choi, W. S., Choi, Y., \& Kang, D. 2012, Highly Reliable Wireless Location Awareness Infra Technology, KGS2012, 235-236

Kim, D. K. \& Choi, W. S. 2011, WLAN RTT-based positioning technology for LBS, TTA Journal, 134, 96-102

Kim, H. 2006, Wireless LAN-Based LBS Services, Telecommunications Review, 16, 188-202

Hong, I. 2009 , New Hope of Location-based services, WPS, SW Insight, 36-53

Ergin, M. A. 2007, in Proceedings of the 13th annual ACM MobiCom, Understanding the effect of access point density on wireless LAN performance, 350-353

Choi, W. S., Han, K. Y., Kim, C. W., Song, H. S., Kim, D. K., \& Choi, J. H. 2011, Trends on LBS Standardization, Information and Communications Magazine, 28 , 15-23

Huang, X., Behr, U., \& Wiesbecd, W. 2000, in Proceedings of the IEEE Global Telecommunications Conference, Automatic cell planning for a low-cost and spectrum efficient wireless network, 1, 282-276

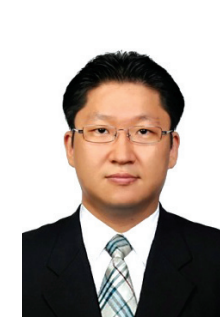

Youngwoo Choi received his B.S., and M.S. degrees in Electronic Engineering from Korea University, Korea, in 1998 and 2000, respectively. He was a researcher since 2007 and is currently working as a senior researcher in Electronics and Telecommunications Research Institute (ETRI). His research interests include ultra-wideband system (UWB), indoor positioning system based RF technology and $\mathrm{RF} /$ analog circuit design.

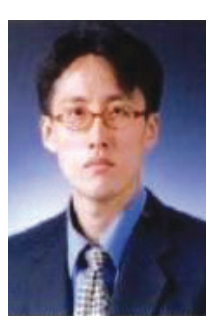

Dong Kyoo Kim received his BS from Kyungpook University and his PhD from Pohang University of Science and Technology, both in electronic engineering, in 1999 and 2005, respectively. He is currently a senior researcher at Electronics and Telecommunications Research Institute, Korea. His research interests include signal processing of positioning, bio, and communication systems. 


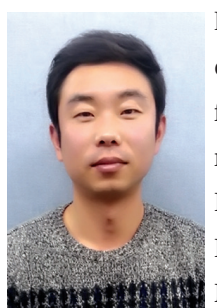

Do Wook Kang received the B.S. and M.S. degrees in Electrical \& Electronic Engineering from Dongshin University in 2002 and 2004, respectively. From 2005 to 2011, he was a Senior Engineer at R\&D Center, Fumate Co., Daejeon, Rep. of Korea, where he developed High-speed joined ETRI, Daejeon, Rep. of Korea. His current research interests include Ground

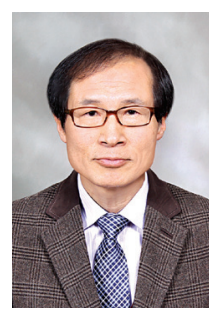

Wan Sik Choi received his BS in Mechanical Engineering from Sung Kyun Kwan University in 1979, MS in Mechanical Engineering, MA in Applied Math, PhD in Mechanical Engineering from the University of Alabama in 1986, 1988, 1992 respectively. He is currently a principal researcher at Electronics and Telecommunications Research Institute (ETRI), Korea. His research interests include optimal control, LBS, GNSS technologies etc. 